\title{
Work-related Burden of Diabetes Mellitus among Employees
}

\author{
Hajar J. Alsalem and Aqil S. Alhaiz
}

\section{ABSTRACT}

Diabetes mellitus (DM) is a major public health problem. The prevalence of DM had increased worldwide despite the availability of medications. Working-age group is mostly affected by DM and its complications which subsequently would affect their quality of life and work productivity. A narrative review of the related literature published in English language from January 2010 to December 2020 using PubMed and Google Scholar databases was performed to explore the burden of DM among employees. There is an increase in absenteeism from work among employees due to DM and its complications, presenteeism was negatively affected. Moreover, employees with DM went through early retirement due to DM and its complications compared to employees without DM. This call to focus on implementing a prevention program to help reduce the impact of DM in employees' work productivity.

Keywords: cost of illness, diabetes mellitus, employee, work productivity.

\author{
Submitted : May 08, 2021 \\ Published : May 31, 2021 \\ ISSN: 2593-8339 \\ DOI: $10.24018 /$ ejmed.2021.3.3.869 \\ Dr. Hajar J. Alsalem* \\ Ministry of Health, Alahsa, Saudi \\ Arabia. \\ (e-mail: hajar.ib84@gmail.com) \\ Dr. Aqil S. Alhaiz \\ Family Medicine Consultant. \\ Ministry of Health, Alahsa, Saudi \\ Arabia. \\ (e-mail: aqilalhaiz1@gmail.com) \\ *Corresponding Author
}

\section{INTRODUCTION}

Diabetes mellitus (DM) is a chronic disease that develops when the pancreas is no longer able to produce insulin, or when the body cannot respond well to the produced insulin [1], [2]. Worldwide, DM is one of the most prevalent diseases threatening the health of individuals. In 2019, DM is estimated to affect 463 million people worldwide and this number is projected to reach 578 million by 2030 . Three out of four people with DM are of working age group (20-64 years). This is equivalent to 351.7 million people and this number is projected to reach 417.3 million in 2030. In 2019, the number of deaths among adults aged 20-79 years which attributed to DM and its complications was 4.2 million. Moreover, DM is estimated to be associated with $11.3 \%$ of deaths globally from all causes among people in this age group. Around $46.2 \%$ of the deaths associated with DM in the working age group occurred in people under the age of 60 years old- working age group [3].

Uncontrolled DM results in complications such as double the risk of cardiovascular events. Diabetes mellitus is also estimated to cause $80 \%$ of chronic kidney diseases. Diabetic eye diseases may lead to blindness with an incidence of $22 \%$ to $33 \%$. Moreover, diabetic foot, which affects 40-60 million people globally, may lead to amputation, which has a large effect on patients' quality of life, may lead to disability, and death [3].

Diabetes mellitus costs can be measured either directly by determining health expenditure or indirectly by assessing the work productivity for an individual suffering from DM or its complications [4], [5]. Globally, the largest health expenditure related to DM is in the age groups of 60-69 years old and 50-59 years old with USD 177.7 billion and 173.0 billion, respectively [3]. The indirect costs related to work productivity are assessed by how the disease will likely affect presenteeism of employees, increase days of absenteeism from work, disability, and early retirement [4], [6], [7].

The effect of DM in people of working age group will add more concern for employees, employers, and society because of the impact in their work productivity. The impact of DM on employees has been observed in cross-sectional as well as prospective studies, with relative consistency across different populations. The studies have concluded that DM has an effect on individuals' quality of life and work productivity [8]-[10]. This review is aimed to summarize the available evidence of the indirect cost of DM and its complications among employees.

\section{METHODS}

PubMed and Google Scholar databases were systematically searched for relevant studies published between January 2010 and August 2020 with no language restriction. Original researches, systematic reviews, and meta-analyses were included. The selection of studies was in three stages: in the primary screening stage, an electronic search was conducted using the following terms: "(cost of illness OR productivity OR presenteeism OR absenteeism OR early retirement) AND (DM) AND (employees)." Article title and abstracts were then screened. The secondary 
screening stage involved the assessment of the full-text our eligibility criteria were extracted. Table I summarize manuscripts. Finally, In the inclusion stage, studies that met these studies.

TABLE I: SUMMARY OF THE PRIMARY STUDIES INCLUDED IN THE REVIEW

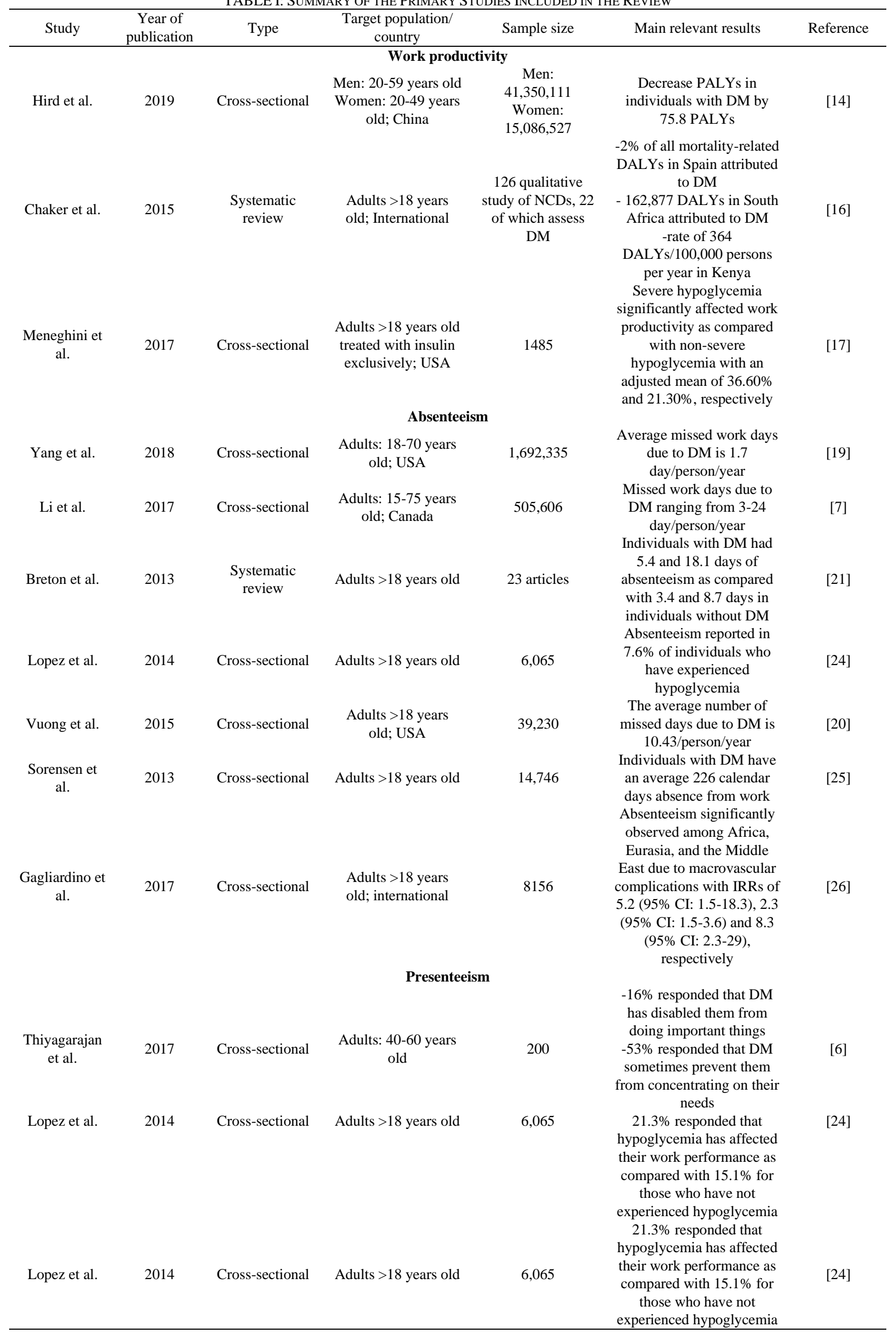




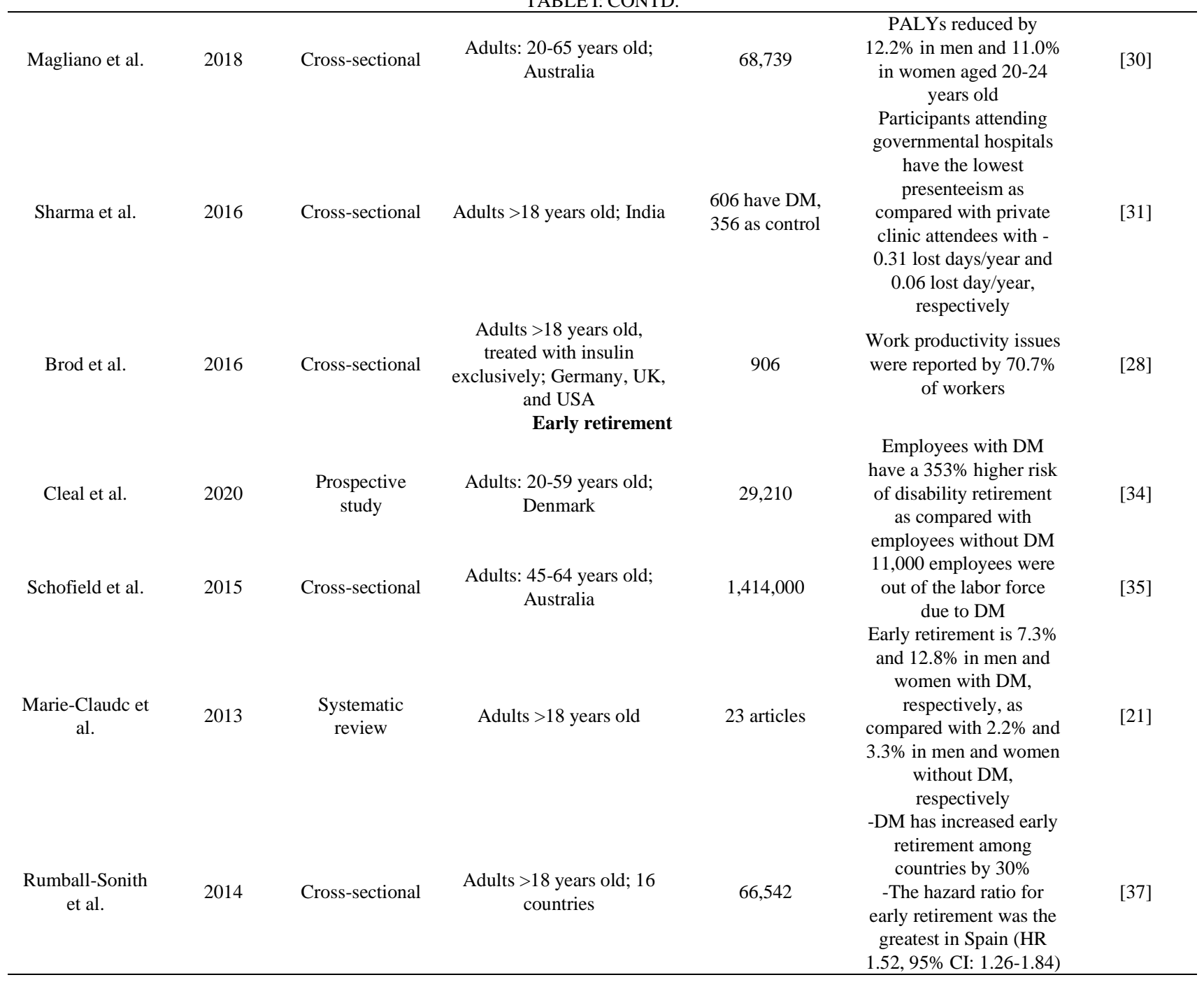

\section{REVIEWING FINDINGS}

\section{A. Work Productivity}

Productivity is defined as a ratio between output measurement and input measurement that differs according to the work itself, the employee, or the time of the work. ${ }^{11}$ Work productivity has been assessed in several studies arguing that DM and its complications have both direct and indirect effects on individuals' productivity at work [12], [13]. One study on Chinese populations uses labor force participation information taken from the International Labor Organization (ILO) for the year 2017. This study includes 41,350,111 men aged between 20 to 59 years old and $15,086,527$ women aged between 20 to 49 years old, and the participants were followed for one year. The results show that the prevalence of DM in the working-age group was $7.1 \%$. Furthermore, they used productivity-adjusted life years (PALYs) to measure the adjusted years of life lived with productivity loss due to DM among employees. There was a total of 75.8 PALYs that decreased among employees with DM, with an average of 1.3 PALYs per person [14]. The burden of DM has also been quantified by measuring the disability-adjusted life years (DALYs) that are used to measure the number of years lived with disability or early death to have an estimate about healthy life lost due to the disease [15]. On the global level, Chaker et al. provide a systematic review to assess the impact of six noncommunicable diseases (NSDs), including DM, on individuals' work productivity. Their review includes 126 studies, 22 of which address DM. They conclude that $2 \%$ of all mortality-related DALYs in Spain were attributed to DM. Moreover, the authors have reported that annually DM had contributed to 162,877 DALYs and 364 DALYs per 100,000 in South Africa and Kenya, respectively [16].

In the US context, Meneghini et al. used the work productivity and activity impairment (WPAI) questionnaire to assess the effect of hypoglycemia in employees treated exclusively with insulin. Approximately 3,758 respondents are included in the study, and they are categorized as not having experienced hypoglycemia in the last three months (938), having experienced non-severe hypoglycemia (1335), and having experienced severe hypoglycemia (1485). The authors conclude that the respondents who have experienced severe hypoglycemia show greater impairments in their work productivity with $36.60 \%$ as compared with $21.30 \%$ in respondents who have experienced non-severe hypoglycemia [17], [18].

Work productivity has been assessed in the above- 
mentioned studies through different methods and among different populations. We can conclude that DM indirectly could affect work productivity.

\section{B. Effect of DM in Presenteeism}

Presenteeism is defined as the ability to finish tasks on time while at work [19]. Several studies have been conducted to assess this theme of indirect cost of DM and show that DM is associated with decreased presenteeism of affected employees. A cross-sectional study conducted through a selfadministered questionnaire among 200 individuals with DM aged 40-60 years old. The authors include subjects diagnosed with DM for the last three years without any diabetic complications. Approximately $16 \%$ of subjects reported that the disease had disabled them from doing important tasks. Among respondents, $45 \%$ had trouble in getting up and being active in the early morning, which has an effect on their presenteeism at work [6].

Hypoglycemia is one of the side effects of hypoglycemic medications, which may affect the presenteeism of employees. Lopez et al. conducted a study that assesses the impact of hypoglycemia on patients with type- 2 diabetes. The results show that hypoglycemia had affected the work performance in $21.3 \%$ of the individuals as compared to $15.1 \%$ of those who did not experience hypoglycemia [24].

The association between post-prandial hyperglycemia (PPH) and presenteeism has been addressed in a web-based survey for Germany, the UK, and the US. The authors include 906 participants who are diagnosed with DM and are on exclusive insulin treatment, to assess PPH and its effect on their work. Approximately $66.6 \%$ of the US participants responded that they experienced PPH during the last week, as compared with $63 \%$ and $53.4 \%$ for German and UK participants, respectively. Among workers, $70.7 \%$ reported an effect on work performance due to PPH. Making more mistakes at work were reported by $27.8 \%, 54.4 \%$ complained of concentration difficulties, and $44.5 \%$ responded that they were less productive at work due to PPH. Moreover, $77 \%$ of respondents with type-2 DM reported that they have decreased work performance ability as compared with $62.9 \%$ of type-1 DM respondents [28].

These results are supported by a study conducted by Magliano et al. in which PALYs lost is used to estimate diabetes burden. The authors include Australian adults aged 20-65 years old who had been diagnosed with diabetes and were registered in the National Diabetes Services Scheme (NDSS) in 2011 [29]. The authors conclude that diabetes reduced PALYs by $11.6 \%$ in men and $10.5 \%$ in women. The impact of diabetes on work productivity is mostly observed among young adults 20-24 years old. PALYs was reduced by $12.2 \%$ in men and $11.0 \%$ in women [30]. Another study conducted by Sharma et al. includes Indians aged more than 18 years old in the period between January and May 2010 to assess the burden of DM on employees. There were 606 participants selected from three clinics: a government-funded tertiary care urban clinic, a private tertiary care urban clinic, and a private primary care rural clinic. The controls are 356 participants who were selected from the local community. The authors conclude that, on an annual basis, 8.8 workdays were lost for government-funded attendees as compared with
44.1 and 43.6 workdays lost for private and rural attendees, respectively. Moreover, the lowest presenteeism is seen for government-funded attendees (- 0.31 days per year), and the highest presenteeism is seen for private attendees $(0.06$ days per year) [31].

We can conclude that uncontrolled DM, side effects of hypoglycemic medications, and the flexibility of appointments all significantly affect individual presenteeism at work.

\section{Absenteeism}

Absenteeism is the number of workdays that are missed due to the poor health of an employee [19]. It has been addressed in several studies among different populations. The included studies had concluded that absenteeism among employees with DM is increased compared to employees without DM. Two cross-sectional studies in the USA support these findings by using national health interview survey (NHIS) data [20]. In the first study, the authors include patients with DM aged 18 to 68 years old. They concluded that the patients had statistically higher missed workdays. There were 1 to 4.2 missed days per year attributed to DM, with an average of 1.7 , and the cost of missed days is estimated to be 3.3 billion dollar per year [19]. In second study, the authors assess the impact of seven common chronic diseases, including DM, in US workers. They include 39,230 workers, 2,563 of which had reported that they have some limitations in their work performance and productivity due to one of the seven chronic diseases. Diabetes mellitus had contributed to an average of 10.43 days lost from work per person annually [20].

$\mathrm{Li}$ et al. conducted a cross-sectional study to measure the impact of DM on occupational health in Canada. The data was obtained from a survey conducted by the national statistical office named, Statistics Canada. They include a representative sample of people aged above 12 years old from the general Canadian population. The survey was conducted every two years since 2001 with a positive association between diabetes and missed workdays which shown in 2010 cohort. The workdays missed that are attributed to diabetes or its complications ranged from 3 to 24 days per year [7]. This result is supported by a systematic review that includes 23 studies. Eight of these studies address absenteeism and conclude that individuals with diabetes had 5.4 to 8.1 days of absenteeism as compared with 3.4 to 8.7 days for individuals without diabetes [21].

Hypoglycemia is one of the most common complications of DM that may affect individuals' daily lives and lead to death [22]. Lopez et al. conducted a cross- sectional study among the US adult population aged over 18 years old. The participants were recruited from the US national health and wellness survey (NHWS), which is an internet-based survey [23]. It consists of a self-administered questionnaire with 41 questions about diabetes. Participants were asked if they take any hypoglycemic medications, if they had ever experienced a hypoglycemic attack within the previous three months, or if they never experienced a hypoglycemic attack. 6,065 participants were included, and $7.6 \%$ of them responded that they were absent from their work for the past three months due to a hypoglycemic attack. In contrast, only $4.4 \%$ of participants who have never experienced hypoglycemia 
noted absenteeism [24].

Sorensen et al. conducted a study among workers diagnosed with DM aged 18-70 years old to assess the impact of DM complications. They obtained individuals' data from the National Patient Registry (NPR) and social service statistics for the period 1997-2002. They include 33,719 workers, $28 \%$ of whom went through early retirement due to DM complications. The average absent days from work was approximately 226 day, which corresponds to $61 \%$ of a year per individual [25]. Moreover, the International Diabetes Management Practice Study (IDMPS), which is conducted every ten years to assess health resources use (HRU) and the impact of DM, supports this result. This study selects physicians randomly and asks them to select every 10 patients diagnosed with DM when attending their clinic to follow-up for two weeks. In this review, the author uses the data from 2011 and 2012. This data includes 8156 patients from 18 countries. The authors conclude that macrovascular complications significantly affect absenteeism rates in Africa, Eurasia, and the Middle East. The incidence rate ratios (IRRs) for each population were 5.2 (95\% CI: $1.5-$ 18.3), 2.3 (95\% CI: $1.5-3.6$ ) and 8.3 (95\% CI: $2.3-29)$, respectively [26].

\section{Early Retirement}

Early retirement is a situation in which a person stops working earlier than the usual statutory retirement age [31]. Diabetes and its complications have been associated with early retirement [33]. One example of a recent study that reports this association is Cleal et al.'s cross-sectional study among employees with DM aged 20-59 years old in Denmark. All participants were registered in the Danish National Diabetes Registry (DNDR) from $1^{\text {st }}$ January 2001 to 30 December 2010. This study includes 29,210 employees and concludes that employees with DM had significantly increased risks of early retirement as compared with employees without DM by $353 \%$ [34].

Another systematic review focuses on adult employees aged 18 years old and above who have been diagnosed with DM. The review includes 23 studies, three of which address early retirement. They conclude that employees with DM stop working early because of their illness as compared with employees without DM. Approximately $7.3 \%$ of men and $12.8 \%$ of women with DM retired early as compared with $2.2 \%$ of men and $3.3 \%$ of women without DM [21].

Deborah Schofield et al. also support these results by using data from the Australian Bureau of Statistics (ABS) survey of disability and ageing and careers (SDACs) from 2003 to 2009 for the 45-64 years old age group. Of the 1,414,000 employees with DM, 11,000 of them stated that they are not in the labor force due to DM [35], [36]. These findings have also been supported by Smith et al.'s study which was conducted among sixteen countries. Data was obtained from the survey of health, ageing, and retirement in Europe (SHARE), the English longitudinal study of ageing (ELSA), and the US health and retirement survey (HRS). The authors include 66,542 participants above 18 years old and diagnosed with DM. Multivariate analysis and the cox proportional hazard ratio are used to compare the risk of early retirement among individuals with DM. The authors conclude that DM has significantly increased the risk of early labor-force exit among nine countries by $30 \%$. The greatest hazard for early labor-force exit among individuals with DM is seen in Spain (HR 1.52; 95\% CI: 1.26, 1.84). The hazard ratios for early labor- force exit of individuals with DM among HRS, ELSA and SHARE participants were 1.40 (95\% CI: 1.30, 1.50), 1.14 (95\% CI: .94, 1.39), and 1.30 (95\% CI:1.23, 1.38), respectively [37].

Early labor force exit has been observed among employees with DM. The association, however, was more prevalent in certain countries, and there was a difference in the association between both sexes.

\section{CONCLUSION}

Diabetes mellitus imposes a considerable burden on individuals, families, and communities. Several studies have shown that DM and its complications had contributed to decrease employees' performance and overall productivity. This review has included 19 study that investigate the indirect cost of DM among employees. The included studies were using different methods in measuring work ability outcome so, the comparability of the results was limited.

The results of this review provide a comprehensive evidence in the indirect cost of DM among employees. This will help employers, insurers, and decision makers to pay attention for the ability to work among employees with DM. The employers can provide various educational and preventive services at workplace for employees. This service could work efficiently to support employees by including individuals, personal resources, and the workplace overall environment. The employers could also provide medical insurance services for their employees to cover the management of the disease with better outcome in their work productivity. The health care professionals could discuss the work productivity issues with their patients to motivate them to follow the management plan of their DM to improve their ability to work(38).

\section{STATEMENT OF ETHICS}

The study is exempt from ethics committee because it is based on review of previously published literature.

\section{CONFLICT OF INTEREST}

The authors have no conflicts of interest to declare.

\section{FUNDING SOURCES}

The authors received no specific funding for this work.

\section{REFERENCES}

[1] Care M. Standards of Medical Care in diabetes - 2020. 2020; 43 (January).

[2] Kelly J. Diabetes: What is diabetes ? CDC Natl Cent Chronic Dis Prev Heal Promot. 2011; 1-3.

[3] Atlas IDFD. Idf diabetes atlas. 2019.

[4] Bonsdorff MB Von, Bonsdorff ME Von, Haanpää M, Salonen M, Mikkola TM. Work - loss years among people diagnosed with diabetes : a reappraisal from a life course perspective. Acta Diabetol 
[Internet]. 2018; 55(5):485-91. Available from: https://doi.org/10.1007/s00592-018-1119-x.

[5] Png ME, Yoong J, Phan TP, Wee HL. Current and future economic burden of diabetes among working-age adults in Asia : conservative estimates for Singapore from 2010-2050. BMC Public Health [Internet]. 2016;1-9. Available from: http://dx.doi.org/10.1186/s12889-016-2827-1.

[6] Thiyagarajan S, John S. Does diabetes blight work productivity: A cross-sectional investigation on selected Type 2 diabetes participants. 2017;43-6.

[7] Article O. Impact of Diabetes Mellitus. 2017;8:96-108.

[8] Data from the GAZEL cohort study. 2011;34(March).

[9] Kirigia JM, Sambo HB, Sambo LG, Barry SP. Economic burden of diabetes mellitus in the WHO African region. BMC Int Health Hum Rights. 2009;9(1):1-12.

[10] Langley P, Stankus A, Annunziata K, Gross H. Pdb37 the Impact of Diabetes on Workplace Absenteeism and Presenteeism: a Comparison of China and Japan. Value Heal. 2010;13(3):A61.

[11] Sauermann J. Performance measures and worker productivity. IZA World Labor. 2016;(May):1-11.

[12] Sadosky A, Schaefer C, Mann R, Bergstrom F, Baik R, Parsons B, et al. Burden of illness associated with painful diabetic peripheral neuropathy among adults seeking treatment in the US: Results from a retrospective chart review and cross-sectional survey. Diabetes, Metab Syndr Obes Targets Ther. 2013;6:79-92.

[13] Sylvia M. Work limitations and their relationship to morbidity burden among academic health center employees with diabetes. Reply. Workplace Health Saf. 2013;61(2):55.

[14] Hird TR, Zomer E, Owen A, Chen L, Ademi Z, Magliano DJ, et al. The impact of diabetes on productivity in China. Diabetologia. 2019;62(7):1195-203.

[15] Donev D, Lijana ZK, Burazeri G. Measuring burden of disease: Disability-Adjusted Life Years ( DALY ) MEASURING THE BURDEN OF DISEASE : 2013;(December):2-3.

[16] Chaker L, Falla A, van der Lee SJ, Muka T, Imo D, Jaspers L, et al. The global impact of non-communicable diseases on macro-economic productivity: a systematic review. Eur J Epidemiol [Internet]. 2015;30(5):357-95. Available from: http://dx.doi.org/10.1007/s10654-015-0026-5.

[17] Meneghini LF, Lee LK, Gupta S, Preblick R. Association of hypoglycaemia severity with clinical, patient-reported and economic outcomes in US patients with type 2 diabetes using basal insulin. Diabetes, Obes Metab. 2018;20(5):1156-65.

[18] English US. WPAI:GH V2.0 (US English) 1. 0:6-7.

[19] S U. Economic Costs of Diabetes in the. 2018;41(May):917-28.

[20] Guang-Wei Zhang, Li Shen2,3, Wen Zhong2, Ying Xiong1,*, Li I. Zhang2,3,*, and Huizhong W. Tao2,3,*. 乳鼠心肌提取 HHS Public Access. Physiol Behav. 2016;176(1):139-48.

[21] Uénette LINEG. Burden of Diabetes on the Ability to. 2013;36.
[22] Shafiee G, Mohajeri-tehrani M, Pajouhi M, Larijani B. The importance of hypoglycemia in diabetic patients. 2012;11(1):1.

[23] Insights PH. NATIONAL HEALTH AND. 2019.

[24] Annunziata K, Bailey RA, Rupnow MFT. Impact of hypoglycemia on patients with type 2 diabetes mellitus and their quality of life, work productivity , and medication adherence. 2014;683-92.

[25] Sørensen J, Ploug UJ. The Cost of Diabetes-Related Complications Registry-Based Analysis of Days Absent from Work. 2013;2013.

[26] Gagliardino JJ, Atanasov PK, Chan JCN, Mbanya JC, Shestakova M V., Leguet-Dinville P, et al. Resource use associated with type 2 diabetes in Africa, the Middle East, South Asia, Eurasia and Turkey: Results from the international diabetes management practice study (IDMPS). BMJ Open Diabetes Res Care. 2017;5(1):1-10.

[27] Rebolledo OR, Dato SMA. Postprandial hyperglycemia and hyperlipidemia- generated glycoxidative stress : its contribution to the pathogenesis of diabetes complications. 2005;(Figure 1):191-208.

[28] Brod M, Nikolajsen A, Weatherall J, Pfeiffer KM. The Economic Burden of Post-prandial Hyperglycemia (PPH) Among People with Type 1 and Type 2 Diabetes in Three Countries. Diabetes Ther. 2016;7(1):75-90.

[29] Form AT. Registration Form. 2008;(1300)

[30] Magliano DJ, Martin VJ, Owen AJ, Zomer E, Liew D. The Productivity Burden of Diabetes at a Population Level. 2018;41(May):979-84.

[31] Sharma KM, Ranjani H, Zabetian A, Datta M, Deepa M, Anand Moses $\mathrm{CR}$, et al. Excess cost burden of diabetes in Southern India: A clinicbased, comparative cost-of-illness study. Glob Heal Epidemiol Genomics. 2016;1:4-11.

[32] Dorn D, Sousa-Poza A. "Voluntary" and "involuntary" early retirement: An international analysis. Appl Econ. 2010;42(4):427-38.

[33] De Wind A, Geuskens GA, Reeuwijk KG, Westerman MJ, Ybema JF, Burdorf A, et al. Pathways through which health influences early retirement: A qualitative study. BMC Public Health. 2013;13(1).

[34] Cleal B, Poulsen K, Hannerz H, Andersen LL. A prospective study of occupational status and disability retirement among employees with diabetes in Denmark. 2009;1:8-10.

[35] Schofield D, Cunich M, Kelly S, Passey ME, Shrestha R, Callander E, et al. The Impact of Diabetes on the Labour Force Participation, Savings and Retirement Income of Workers Aged 45-64 Years in Australia. 2015;1-10.

[36] Disability, ageing and carers: 2003;(02)

[37] Rumball-Smith J, Barthold D, Nandi A, Heymann J. Diabetes associated with early labor-force exit: A comparison of sixteen highincome countries. Health Aff. 2014;33(1):110-5.

[38] Ruston A, Smith A, Fernando B. Diabetes in the workplace - diabetic' $\mathrm{s}$ perceptions and experiences of managing their disease at work : a qualitative study. 2013;1-10. 